\title{
Effects of hydroxyapatite nanostructure on channel surface of porcine acellular dermal matrix scaffold on cell viability and osteogenic differentiation of human periodontal ligament stem cells
}

This article was published in the following Dove Press journal:

International Journal of Nanomedicine

9 May 2013

Number of times this article has been viewed

\section{Shaohua $\mathrm{Ge}^{\prime}$ \\ Ning Zhao' \\ Lu Wang' \\ Hong Liu \\ Pishan Yang'}

'Shandong Provincial Key Laboratory of Oral Biomedicine, Department of Periodontology, Shandong University; ${ }^{2}$ State Key Laboratory of Crystal Materials, Center of Bio and Micro/Nano Functional Materials, Shandong University, Jinan, People's Republic of China
Correspondence: Hong Liu State Key Laboratory of Crystal Materials, Center of Bio and Micro/ Nano Functional Materials, Shandong University, 27 Shangda Nanlu, Jinan 250100 , People's Republic of China

Tel +86 53। 88362807

Fax +86 53I 88362807

Email hongliu@sdu.edu.cn

Pishan Yang

Key Lab of Oral Biomedicine of Shandong Province, Department of Periodontology, School of Stomatology, Shandong University, 44-I Wenhua Road West, Jinan 2500 I2, People's Republic of China $\mathrm{Tel}+8653188382368$

Fax +86 53I 88382923

Email yangps@sdu.edu.cn
Abstract: A new nanostructured hydroxyapatite-coated porcine acellular dermal matrix (HApPADM) was fabricated by a biomimetic mineralization method. Human periodontal ligament stem cells were seeded on HAp-PADM and the effects of this scaffold on cell shape, cytoskeleton organization, cell viability, and osteogenic differentiation were examined. Periodontal ligament stem cells cultured on HAp-PADM exhibited different cell shape when compared with those on pure PADM. Moreover, HAp-PADM promoted cell viability and alkaline phosphatase activity significantly. Based on quantitative real-time polymerase chain reaction, the expression of bonerelated markers runt-related transcription factor 2 (Runx2), osteopontin (OPN), and osteocalcin $(O C N)$ upregulated in the HAp-PADM scaffold. The enhancement of osteogenic differentiation of periodontal ligament stem cells on the HAp-PADM scaffold was proposed based on the research results. The results of this study highlight the micro-nano, two-level, three-dimensional HAp-PADM composite as a promising scaffold for periodontal tissue engineering.

Keywords: hydroxyapatite, scaffold, nanostructure, proliferation, differentiation, tissue engineering

\section{Introduction}

As one of the key elements of tissue engineering, scaffolds are used to create the threedimensional (3D) organization for appropriate cell interactions and to serve as vehicles to deliver and retain cells and molecules. The scaffold materials studied widely for periodontal tissue engineering include chitosan, ${ }^{1,2}$ bioactive glass, ${ }^{3}$ and silk. ${ }^{4}$ Among them, porcine acellular dermal matrix (PADM) has been paid much attention because of its good biocompatibility, biodegradation properties, and easiness to prepare. PADM is mainly composed of type I and II collagen and has been successfully used in covering full-thickness burn wounds in clinical practice. ${ }^{5}$ Moreover, a purified porcine skin sheet can be easily tailored to a certain size and shape for a variety of applications. ${ }^{6}$ Recent studies have indicated that surface topology of biomaterials could profoundly affect cell functions, ${ }^{7,8}$ and participate in implanted cell lineage commitment. ${ }^{9}$ A new tendency of scaffold material development is to increase the mechanical stability and to promote osteogenic commitment of cells. The assembly of bone substitute on polymer scaffold could induce seed cells to differentiate towards osteoblasts ${ }^{9,10}$ as well as increase mechanical strength. ${ }^{9}$ As one kind of bone substitute, hydroxyapatite 
(HAp) has been widely used for bone regeneration and dental implant osseointegration because of its osteoconductive and osteoinductive properties..$^{11,12}$

Currently, by a biomimetic mineralization method, the authors' group and Guo et al successfully assembled HAp on the channel surface of PADM, and prepared a bioactive scaffold for periodontal tissue engineering (HAp-PADM). ${ }^{6,13}$ It was found that HAp-PADM had relatively high mechanical strength and reduced the high rate of enzymatic degradation of natural collagen, which could increase the compressive resistance and stabilize the collagen-based materials. Moreover, the HAp-PADM scaffold was biocompatible for human periodontal ligament (PDL) cells and MC3T3-E1 preosteoblasts, and the cells were able to proliferate and migrate into the scaffold. ${ }^{6,13}$ Therefore, this composite system combining the advantages of PADM and HAp seems to be a promising choice for periodontal tissue regeneration.

In this study, human PDL stem cells (PDLSCs) were cultured and identified, and then seeded on HAp-PADM and pure PADM scaffolds. The morphology and distribution of PDLSCs were examined with scanning electron microscope (SEM) and confocal laser scanning microscope (CLSM). In addition, methylthiazol tetrazolium (MTT) assay was used to evaluate cell viability, and alkaline phosphatase (ALP) activity was detected to determine osteogenic differentiation of PDLSCs on the scaffolds. Moreover, the expression of bone-related markers runt-related transcription factor 2 (Runx2), osteopontin (OPN), and osteocalcin (OCN) were detected by reverse transcriptase polymerase chain reaction (RT-PCR).

\section{Material and methods Preparation of PADM and HAp-PADM scaffolds}

A PADM scaffold with natural microporous structure was decellularized through a series of agitations involving enzymes, detergents, and hyperosmotic saline treatment. The obtained PADM is used for preparation of HApPADM scaffolds by assembling HAp nanostructures on the channel surface of a PADM scaffold through a biomimetic chemical mineralization process in simulated body fluid. The preparation process, structural and morphological characterization, mechanical properties, and in vitro biodegradation rate of PADM and HAp-PADM scaffolds were described in detail previously. ${ }^{6}$ PADM is a $3 \mathrm{D}$ interconnected network structure, mainly composed of collagen I, with channel diameter over $100 \mu \mathrm{m}$ and channel wall thickness of about 10-20 $\mu \mathrm{m}$. The HAp-PADM composite scaffold is a two-level, 3D network structure. After the biomimetic mineralization process, the composite still keeps its 3D interconnected network structure, which is the first level of the 3D network structure. A layer of HAp $3 \mathrm{D}$ network nanostructure with about $120 \mathrm{~nm}$ in channel diameter is assembled on the surface of the channel. This special structure endows the HAp-PADM composite scaffold with high strength, long-term biodegradation durability, and osteogenic differentiation promotion potential.

\section{Collection and culture of human PDLSCs}

Written approval for human PDL collection performed in this study was obtained from the Medical Ethics Committee of Medical School, Shandong University (approval number: 2010015; Jinan, People's Republic of China) and written informed consent was obtained from each individual participant. Teeth were collected from clinically healthy premolars extracted for orthodontic reasons. PDLSCs were collected and cultured as previously described. ${ }^{14}$ Briefly, PDL tissues were digested, and dissociated cell suspension was filtered and seeded at a low concentration for single cell-derived colony selection. Individual colonies were isolated with colony rings and expanded into individual vessels for further cultivation.

\section{Immunocytochemical staining}

PDL single-cell colonies were cultured on eight-well chamber slides until confluent and immunocytochemical staining for mesenchymal stem cell (MSC) marker, STRO-1, was performed as previously described. ${ }^{14}$ Briefly, after fixing with $4 \%$ paraformaldehyde and sealing with $10 \%$ bovine serum albumin (Sigma-Aldrich, St Louis, MO, USA), cells were incubated with diluted primary antibody anti-STRO-1 (Santa Cruz Biotechnology, Santa Cruz, CA, USA) or mouse immunoglobulin G1 (IgG1) as a negative control, and finally with fluorescein-conjugated secondary antibody. Afterwards, the cell nuclei were counterstained with 4',6-diamidino-2phenylindole (Sigma-Aldrich). The slides were mounted and then analyzed under a fluorescence microscope (BX51, Olympus Ltd, Tokyo, Japan).

\section{Flow cytometric analysis}

PDLSCs were detached with $0.05 \%$ trypsin/ ethylenediaminetetraacetic acid and resuspended in blocking buffer containing Hanks' balanced salt solution (SigmaAldrich) supplemented with 5\% fetal calf serum, 1\% bovine serum albumin, and 5\% normal human serum for 1 hour. Approximately $1 \times 10^{5}$ cells were incubated with specific fluorescein isothiocyanate-conjugated mouse monoclonal 
antibodies $(10 \mu \mathrm{g} / \mathrm{mL})$ for human cluster of differentiation 44 (CD44), CD73, CD90, CD105, CD166 (Becton Dickinson Biosciences, San Jose, CA, USA), CD14, CD34, CD45 (Beckman Coulter, Brea, CA, USA), or $10 \mu \mathrm{g} / \mathrm{mL}$ isotypematched control 1B5 (IgG1) and 1D4.5 (IgG2a). After incubation on ice for 1 hour, cells were incubated with goat anti-mouse IgG or IgM for 45 minutes on ice. After washing, flow cytometry data were analyzed using an EPICS $^{\circledR}$ XL/ MCL $^{\text {TM }}$ flow cytometer (Beckman Coulter).

\section{PDLSCs cultured on the scaffolds}

PADM and HAp-PADM samples were cut into $8 \mathrm{~mm} \times 8 \mathrm{~mm} \times 0.6 \mathrm{~mm}$ pieces for cell culture. First, the scaffolds were sterilized using $75 \%$ ethanol for 2 hours in a 24-well plate, exposed to ultraviolet radiation for 30 minutes, washed three times with sterile phosphate buffered saline, and then transferred into 24-well culture plates and prewetted with $\alpha$-minimal essential medium overnight. After $80 \%$ confluence, PDLSCs were digested with $0.25 \%$ trypsin, and $100 \mu \mathrm{L}$ cell suspension containing $5 \times 10^{4}$ cells were seeded into each scaffold. After 3 hours, another $900 \mu \mathrm{L}$ culture medium was added to each well, and the culture was maintained at $37^{\circ} \mathrm{C}$ in $5 \%$ carbon dioxide and $95 \%$ humidity.

\section{SEM observation}

PDLSCs morphology on HAp-PADM and PADM scaffolds was examined using an SEM (S-750; Hitachi Ltd, Tokyo, Japan). The detailed method has been described elsewhere. ${ }^{14}$

\section{CLSM observation}

PDLSCs $\left(5 \times 10^{4}\right)$ were seeded into each scaffold in 24-well plastic culture plates. Forty-eight hours after initial seeding, the cell-coated samples were fixed with 3.7\% formaldehyde solution for 10 minutes and then extracted with $0.1 \%$ Triton X-100 (Sigma-Aldrich) for 5 minutes and blocked with phosphate buffered saline containing $1 \%$ bovine serum albumin (Sigma-Aldrich) for 30 minutes. The samples were then stained with phalloidin conjugated to Alexa Fluor ${ }^{\circledR} 488$ (Life Technologies, Carlsbad, CA, USA) and examined with a TCS SP ${ }_{2}$ CLSM (Leica Microsystems, Wetzlar, Germany).

\section{Cell viability and ALP activity assay}

Cell viability was determined by MTT (3-[4,5-dimethylthiazol2-yl]-2,5-diphenyltetrazolium bromide) assay and ALP activity was measured by the p-nitrophenyl phosphate method as previously described. ${ }^{14}$

\section{Quantitative RT-PCR analysis of bone-related markers}

Osteogenic gene expression of human PDLSCs cultured on HAp-PADM and PADM was explored by RT-PCR analysis as previously described. ${ }^{14}$ Briefly, the cell-seeded HAp-PADM and PADM scaffolds were rinsed after 3, 7, and 14 days, and the cells on the scaffolds were digested and lysed with TRIzol ${ }^{\circledR}$ Reagent (Life Technologies), followed by reverse transcription using Oligo (dT) primers and SuperScript ${ }^{\mathbb{B}}$ III Reverse Transcriptase according to the manufacturer's instructions (Life Technologies). RT-PCR was performed with $\mathrm{RT}^{2} \mathrm{SYBR}^{\circledR}$ Green qPCR Master Mix (SABiosciences, Frederick, MD, USA). The primer sets used (Runx2, OPN, and $O C N$ ) are shown in Table 1. Expression of $\beta$-actin was used as an internal control.

\section{Statistical analysis}

Results are presented as the mean \pm standard deviation of three to nine replicates for each experiment. The statistically significant differences between groups were assessed by one-way analysis of variance. $P<0.05$ was considered statistically significant.

\section{Results \\ Characteristics of PDLSCs}

PDLSCs were identified as single-cell colonies generated from human PDL-derived cells (Figure 1A). This colonyforming cell population is termed as PDLSCs. Cultured PDLSCs exhibit positive staining for MSC marker STRO-1 (Figure 1B), which is originally identified as a marker for colony-forming osteogenic precursor cells isolated from bone marrow. ${ }^{15}$ Moreover, PDLSCs were positive (defined as $\geq 95 \%$ positive cells) for MSC-associated surface markers CD44, CD73, CD90, CD105, and CD166, and were negative ( $\leq 5 \%$ positive cells) for hematological markers CD14, CD34, and CD45 (Figure 2).

Table I Primer sequences for osteogenic markers

\begin{tabular}{lllll}
\hline Gene & GenBank number & Product size (bp) & Forward primer $\mathbf{5}^{\prime} \mathbf{- 3}^{\prime}$ & ${\text { Reverse primer } \mathbf{3}^{\prime} \mathbf{- 5}}^{\mathbf{\prime}}$ \\
\hline Runx2 & NM_001024630 & 137 & GTGGACGAGGCAAGAGTTTCA & CATCAAGCTTCTGTCTGTGCC \\
OPN & NM_001040060 & 92 & GCAGACCTGACATCCAGTACC & GATGGCCTTGTATGCACCTTC \\
OCN & NM_199173 & 255 & ATGAGAGCCCTCACACTCTCG & GTCAGCCAACTCGTCACAGTCC \\
\hline
\end{tabular}

Abbreviations: BP, base pair; OPN, osteopontin; OCN, osteocalcin. 


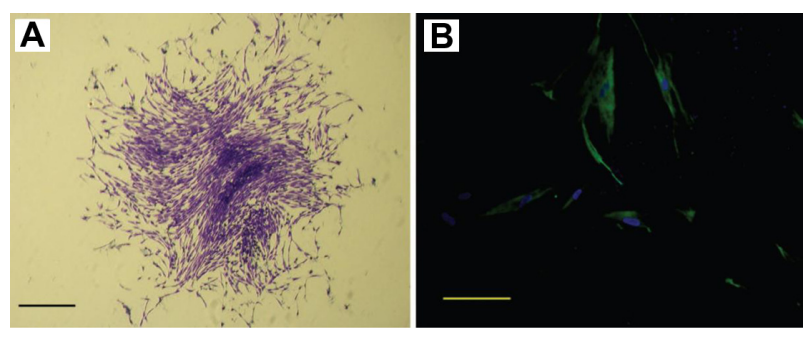

Figure I Characterization of human periodontal ligament stem cells. (A) Cell clusters derived from the periodontal ligament formed a single colony and were stained with $0.1 \%$ toluidine blue (scale bar: $100 \mu \mathrm{m}$ ). (B) Cultured periodontal ligament stem cells exhibited positive staining for STRO-I (green fluorescent signals; blue signals nuclei) (scale bar: $50 \mu \mathrm{m}$ ).

\section{SEM images of PDLSCs on scaffolds}

SEM images of PADM and HAp-PADM are shown in Figure 3. PADM possesses a 3D porous structure with a channel diameter about 50-100 $\mu \mathrm{m}$ (Figure 3A). After the decellularization process, collagen in PADM still keeps its natural structure (Figure 3A). After the biomimetic mineralization process, HAp-PADM keeps the microstructure of PADM and forms a layer of an HAp 3D interconnected nanostructure with 120-150 nm microchannels on the surface of the microchannels of PADM (Figure 3D). Adhesion of human PDLSCs on the two scaffolds after 2 days cell seeding was also observed under SEM. Before being seeded on scaffolds, PDLSCs showed typical fibroblastlike morphology. However, PDLSCs adopt a polygonal shape on HAp-PADM (Figure 3E) while those on pure PADM maintain their intrinsic spindle shapes (Figure 3B) under SEM. PDLSCs adhered on the two scaffolds, and the morphologies of PDLSCs on the two scaffolds are different. On PADM, the vast majority of human PDLSCs appear confluent and exhibit a spindle shape, fibroblast-like morphology and possess the typical phenotype of PDLSCs (Figure 3B). In contrast, the shape of PDLSCs on HApPADM is polygonal and similar to an osteoblast phenotype (Figure 3E). Two cells spread completely on PADM, overlap each other, and adapt very closely to the underlying collagen fibers (Figure 3C), while HAp clusters can be observed on
CD44

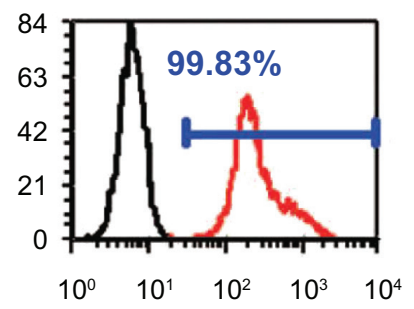

CD73

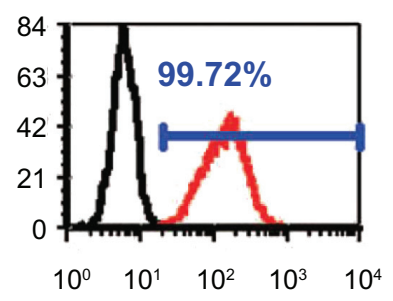

CD166

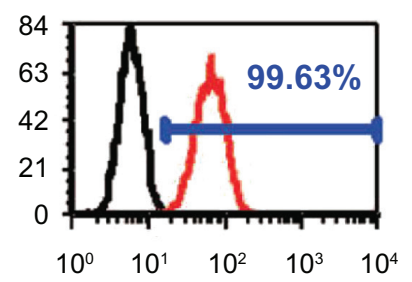

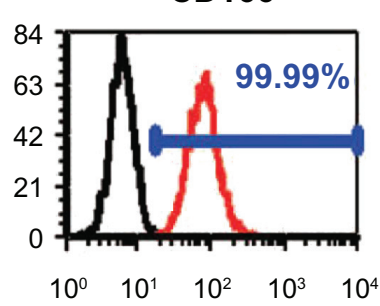

CD34

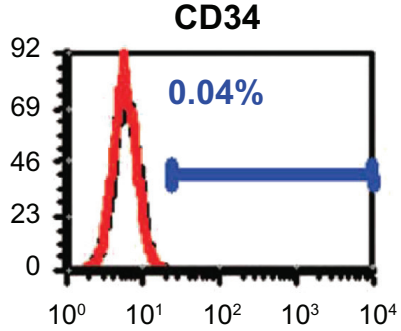

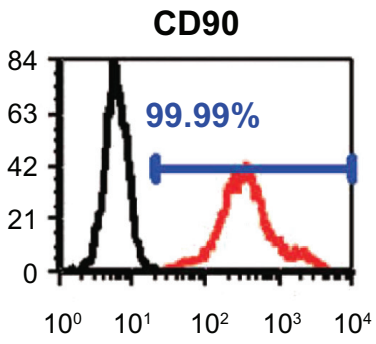

CD14

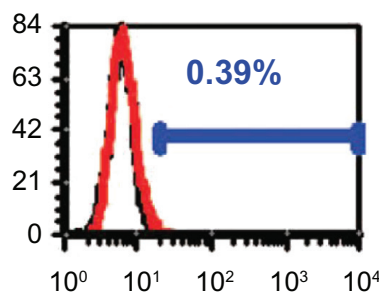

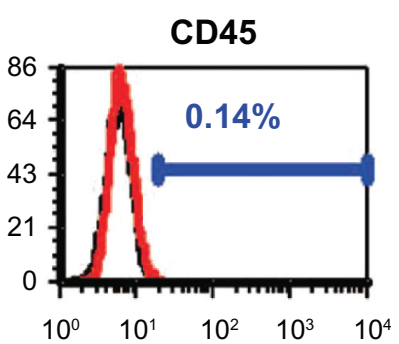

Figure 2 Flow cytometric analysis of periodontal ligament stem cells.

Notes: They were positive for mesenchymal stem cell markers CD44, CD73, CD90, CDI05, and CDI66 and negative for hematopoietic markers CDI4, CD34, and CD45 (red line). The black line represents the isotype controls IB5 (immunoglobulin GI) and ID4.5 (immunoglobulin G2a).

Abbreviation: $C D$, cluster of differentiation. 


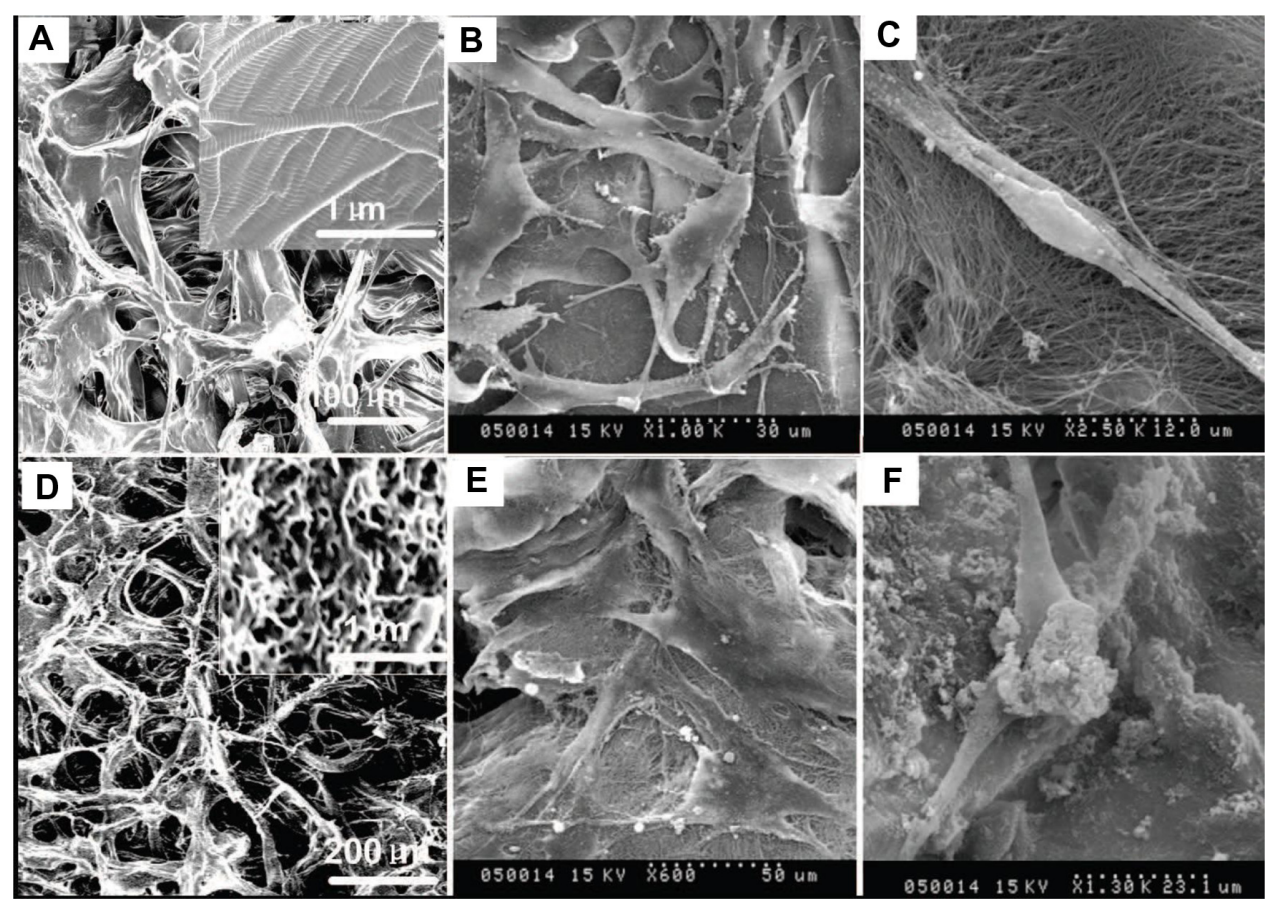

Figure 3 Scanning electron microscope images of human periodontal ligament stem cells on a pure porcine acellular dermal matrix and hydroxyapatite-coated porcine acellular dermal matrix. (A) The porcine acellular dermal matrix possesses a three-dimensional porous structure with channel diameter of about $50-100 \mu$ m. (B) Periodontal ligament stem cells adhered on the porcine acellular dermal matrix uniformly and exhibited typical fibroblast-like morphology. (C) Periodontal ligament stem cells on the porcine acellular dermal matrix extended completely and intimately adhered to the underlying collagen fibrils. (D) A hydroxyapatite three-dimensional interconnected nanostructure with I20-150 nm microchannels formed on the surface of the microchannels of the porcine acellular dermal matrix. (E) Periodontal ligament stem cells exhibited osteoblast-like morphology on the hydroxyapatite-coated porcine acellular dermal matrix. (F) Hydroxyapatite clusters precipitated on the cells and collagen fibers after cells seeding on the hydroxyapatite-coated porcine acellular dermal matrix scaffold.

both cells and collagen fibers 2 days after cell seeding on HAp-PADM (Figure 3F).

\section{CLSM images of PDLSCs on scaffolds}

Morphology and distribution of viable human PDLSCs after 2 days culture on the scaffolds were observed under CLSM to further check the adhesion and immigration of cells on both of the scaffolds. Actin filament was stained by Alexa Fluor 488 phalloidin, which can emit green fluorescence when excited by light with a wavelength of $488 \mathrm{~nm}$. Figure 4A and B show the cell morphology and distribution of viable cells on pure PDAM and HAp-PADM. Cells distribute on the framework with random arrangement due to the natural original channels of the PADM framework. In Figure 4A and B, it can be seen that some fluorescent spots are vague, which indicates that some cells are not on the focal plane. This phenomenon is the typical $3 \mathrm{D}$ growth of the cell, which illustrates that the cells can grow into the channels of the scaffold. The porous surface structure determines the cell position on the scaffold and causes different fluorescence intensity of cells or different parts of one cell to be in and out of focus. From low resolution CLSM images of the samples, it can be seen that cells on PADM seem less dense (Figure 4C) than those on HAp-PADM (Figure 4D).

\section{Cell viability and ALP activity}

There is no statistically significant difference between the pure PADM framework and HAp-PADM scaffolds after seeding for 1 day. However, after 3, 5, and 7 days culture, the viability of cells on HAp-PADM are significantly higher than those on pure PADM $(P<0.05, P<0.01$, and $P<0.01$ respectively; Figure 5). Human PDLSCs exhibited higher viability on HAp-PADM than those on pure PADM. The observation of CLSM agreed with MTT assay, and more cells adhered on the HAp-PADM scaffold.

As an early marker for osteogenesis, ALP is assumed to reflect the degree of differentiation. ALP activity of cells on the two samples measured on days three, seven, and 14 shows that ALP activity peaked after 7 days of cultivation (Figure 6). PDLSCs exhibited higher ALP activity on HApPADM than those on pure PADM on days three and seven ( $P<0.05$ and $P<0.01$, respectively). On day 14, ALP activity decreased and there was no significant difference between the two groups.

\section{RT-PCR analysis of bone-related genes}

Significantly higher expression level of Runx 2 and $O P N$ was found on HAp-PADM than on pure PADM on day seven 

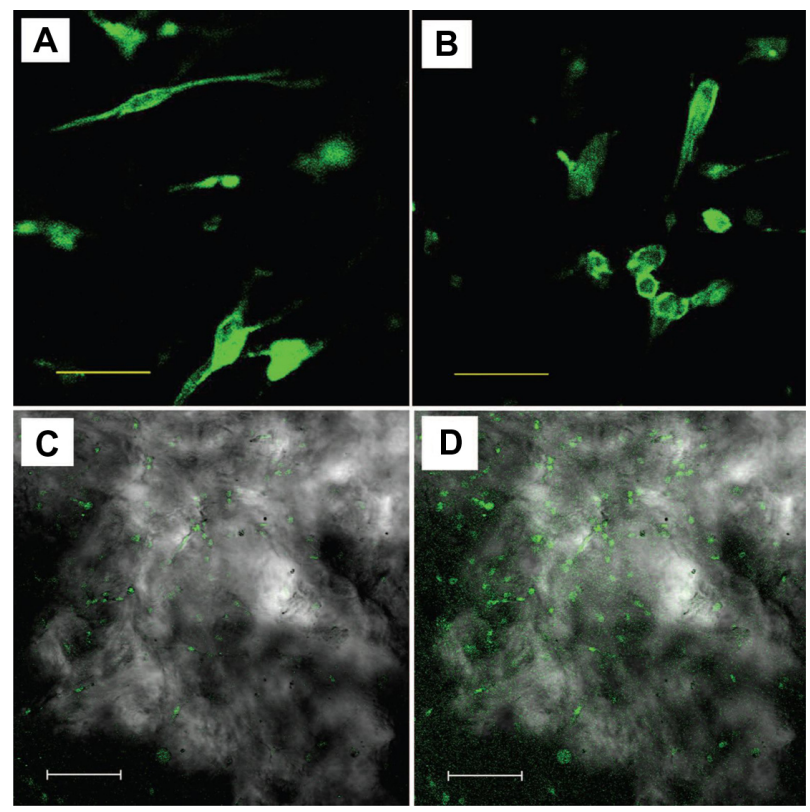

Figure $\mathbf{4}$ Confocal laser scanning microscope images of human periodontal ligament stem cells on a pure porcine acellular dermal matrix and hydroxyapatite-coated porcine acellular dermal matrix at a scale bar of (A and B) $50 \mu \mathrm{m}$ and (C and D) I $50 \mu \mathrm{m}$ after 2 days culture in vitro.

$(P<0.05$; Figure 7), followed by an expression decrease on day 14 with no significant difference at this time point. $O C N$ was continuously upregulated on HAp-PADM and peaked on day 14. The expression level of $O C N$ on HApPADM was significantly higher than that on PADM on day $14(P<0.01)$.

\section{Discussion}

Biomimetic mineralization approaches to assembling a nanostructured apatite layer throughout a $3 \mathrm{D}$ porous bone tissue engineering scaffold have provided an effective tool

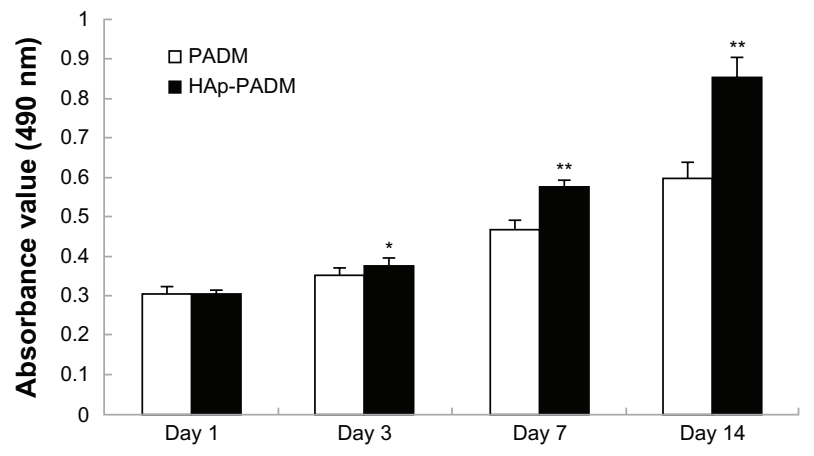

Figure 5 Metabolic activity (methylthiazol tetrazolium assay) of periodontal ligament stem cells seeded on PADM and HAp-PADM.

Notes: Data represents mean \pm standard deviation $(n=9$, nine replicates per time point for each experimental condition); *P $<0.05$; **P $<0.0$ I (PADM versus HApPADM).

Abbreviations: HAp-PADM, hydroxyapatite-coated porcine acellular dermal matrix; PADM, porcine acellular dermal matrix.

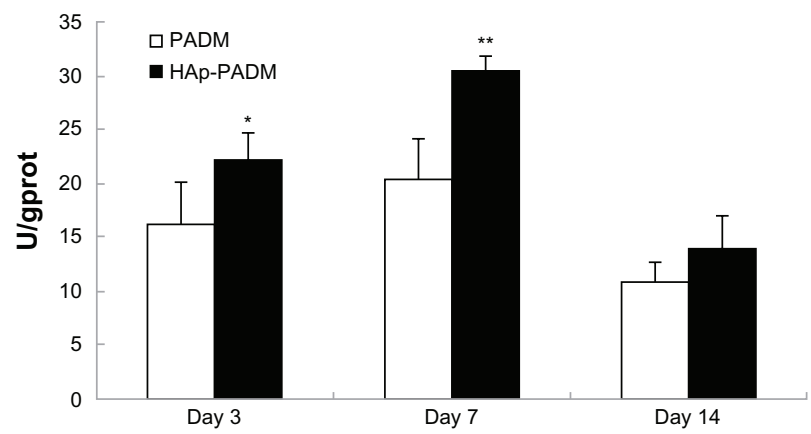

Figure 6 Alkaline phosphatase activity of periodontal ligament stem cells seeded on PADM and HAp-PADM.

Notes: Data were normalized for total protein content to account for the effect of the different number of cells on each scaffold. Data represents mean \pm standard deviation ( $n=5$, five replicates per time point for each experimental condition); $* P<0.05$; **P $<0.0$ I (PADM versus HAp-PADM).

Abbreviations: HAp-PADM, hydroxyapatite-coated porcine acellular dermal matrix; PADM, porcine acellular dermal matrix.

for controlling surface chemistry and geometry within a large and complex structure. ${ }^{9}$ Recently, the authors' group has successfully fabricated an HAp-PADM scaffold with a 3D two-level porous structure by assembling the HAp nanostructures on the channel surface of a PADM framework by a two-step biomimetic mineralization method. This HApPADM scaffold has both interconnected channels $(\sim 100 \mu \mathrm{m}$ in diameter) formed by collagen fiber bundles and nanopores $\left(<100 \mathrm{~nm}\right.$ in diameter). ${ }^{6}$

In this study, the effect of this surface biomimetic apatite nanonetwork structure of PADM on the biological characteristics and osteogenic differentiation potential of PDLSCs was examined. These characteristics were compared with PDLSCs cultured on PADM with a smooth surface.

Before being seeded on scaffolds, PDLSCs showed typical fibroblast-like morphology and expressed MSC markers CD44, CD73, CD90, CD105, and CD166. However, PDLSCs adopt a polygonal shape on HAp-PADM while those on pure PADM maintain their intrinsic spindle shapes under SEM. These results indicate that the surface and chemical composition characteristics of the scaffolds could influence the phenotype of cells. In fact, scaffold mean pore size significantly influences cell morphology and phenotypic expression. ${ }^{16}$ By the assembly of HAp on PADM, the obtained HAp-PADM scaffold has a two-level porous structure with large channels $(\sim 100 \mu \mathrm{m}$ in diameter) inherited from the purified PADM microstructure and small pores (100-120 nm in diameter) made up of self-assembled HAp on the channel surfaces. ${ }^{6}$ The nano-HAp porous structure and calcium element on the channel wall may alter the phenotypic expression of PDLSCs. 

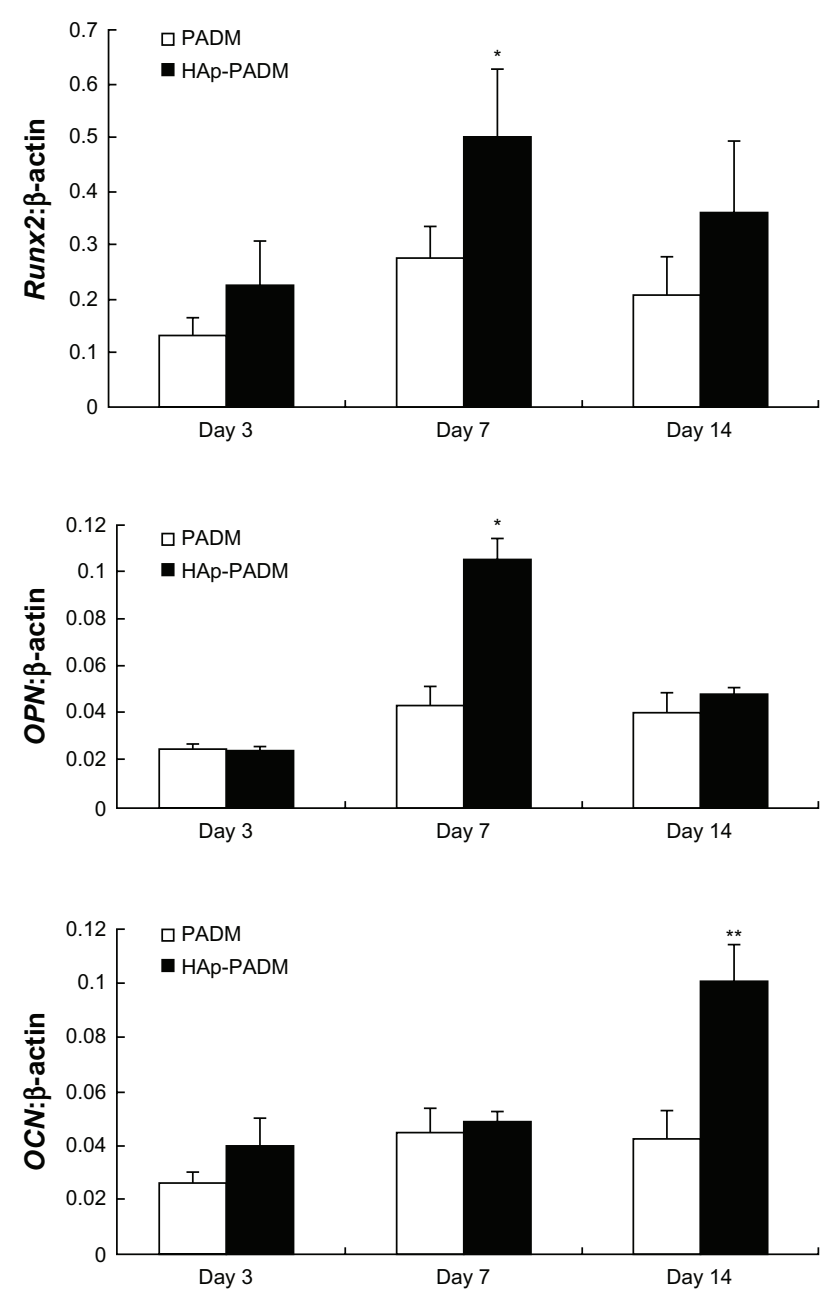

Figure 7 Real-time polymerase chain reaction analysis of Runx2, OPN, and OCN messenger ribonucleic acid expression in periodontal ligament stem cells on two scaffolds.

Notes: Gene expression was normalized to housekeeping gene $\beta$-actin expression. Periodontal ligament stem cells were cultivated for 3, 7, and 14 days on PADM and HAp-PADM. Data represents mean \pm standard deviation $(n=3) ; * P<0.05$; $* * P<0.0$ I (PADM versus HAp-PADM).

Abbreviations: OPN, osteopontin; OCN, osteocalcin; HAp-PADM, hydroxyapatitecoated porcine acellular dermal matrix; PADM, porcine acellular dermal matrix.

The regulation of PDLSC proliferation and viability is crucial for periodontal tissue regeneration. In this study, the proliferative effect of scaffolds on PDLSCs was examined in vitro. Human PDLSCs exhibited higher viability on HApPADM than those on pure PADM. The observation under CLSM agreed with the MTT assay that more cells adhered on HAp-PADM scaffold. Fibroblasts bound to a wide range of pore sizes $(63-150 \mu \mathrm{m})$ and cells increased their viability with decreasing pore size until no cells could fit into the pores. ${ }^{17}$ The pore size of the pure PADM scaffold is about $100 \mu \mathrm{m}$, which is favorable for the attachment and ingrowth of PDLSCs. Moreover, the addition of HAp might increase the scaffold surface area and the small pores made up of the HAp nanostructure may favor the attachment and growth of cells. The promoted effect of HAp-PADM on PDLSCs viability means more pluripotent stem cells can grow on the scaffold, which will facilitate the regeneration of damaged periodontal tissue.

Most importantly, the porous self-assembled 3D continuous network-like nanostructures of HAp might dissolve in culture media in vitro and increase the concentrations of calcium and phosphorus ions. The increase of the concentrations of calcium and phosphorus ions may play an important role in the regulation of cell differentiation. For example, inorganic phosphate has been reported to be a signaling molecule of osteoblast differentiation ${ }^{18}$ and regulates multiple genes during osteoblast differentiation. ${ }^{19}$ As an early marker for osteogenesis, ALP is assumed to reflect the degree of differentiation. In the current study, PDLSCs cultured on HAp-PADM exhibited higher ALP activity than those on pure PADM on days three and seven.

Recent studies demonstrate that synthetically nanofabricated structure can not only affect cell shape, adhesion, migration, and proliferation, but also influence RhoA kinase-mediated cytoskeletal tension, modulation of intracellular signaling pathways that regulate transcriptional level, and gene expression. ${ }^{20,21}$ RT-PCR analysis for osteogenic gene expression further indicates that the surface microenvironment of the scaffolds affects osteogenic differentiation. In the current study, expression of Runx2, $O P N$, and $O C N$ was upregulated on HAp-PADM. Runx2 is a key transcription factor regulating a number of other genes involved in osteoblast differentiation and bone development. ${ }^{22} O P N$ is a multifunctional phosphorylated glycoprotein secreted by osteoblasts at an early stage during bone development to facilitate attachment to the extracellular matrix, ${ }^{23}$ while $O C N$ is synthesized when the remodeling of the bone matrix is activated;22,24 this can explain why the expression of $O P N$ reached its highest level (on day seven) earlier than $O C N$ (on day 14) in this study. The upregulation of Runx2,OPN, and $O C N$ further demonstrates that HAp-PADM can improve osteogenic differentiation of PDLSCs.

Altogether, the results of this study demonstrate that biomimetic HAp nanoconstructs on PADM can alter PDLSCs morphology, increase viability, and significantly enhance PDLSC osteogenic differentiation (increased ALP production and upregulation of bone-related genes). The assembly of the HAp nanostructure might increase the viability and differentiation through two approaches. First, the biomimetic mineralization procedures may change the surface topography and chemistry within the 
porous scaffolds (eg, scaffold surface area, pore diameter). Second, special interface interaction between surface apatite nanostructure and PDLSCs may induce osteogenic commitment. Therefore, further study will explore the biomolecular mechanisms by which nanostructured apatite influence PDLSC biobehavior, and an in vivo experiment with PDLSC-seeded HAp-PADM for repair of periodontal or bone defect may be performed.

\section{Conclusion}

Human PDLSCs were successfully isolated and cultured, and they expressed STRO-1 and were positive for MSC markers while negative for hematological markers. Moreover, when cultured on PADM with surface biomimetic HAp nanostructures, human PDLSCs exhibited a different shape from those cultured on the smooth surfaced PADM. Additionally, HApPADM promoted cell viability, upregulated ALP activity, and induced messenger ribonucleic acid expression of osteogenic differentiation markers of Runx $2, O P N$, and $O C N$. This study demonstrates that the surface HAp nanonetwork structure of PADM could promote the viability and differentiation of PDLSCs and is a promising scaffold for periodontal tissue engineering.

\section{Acknowledgments}

This research was supported by the National Natural Science Foundation of China (Number 81100756, NSFDYS: 5092525), Independent Innovation Foundation of Shandong University (Number 2012TS097), Science and Technology Program of Shandong Province (Number 2010GSF10248). The funders had no role in study design, data collection and analysis, decision to publish, or preparation of the manuscript.

\section{Disclosure}

The authors report no conflicts of interest in this work.

\section{References}

1. Soran Z, Aydin RS, Gumusderelioglu M. Chitosan scaffolds with BMP-6 loaded alginate microspheres for periodontal tissue engineering. J Microencapsul. 2012;29(8):770-780.

2. Akman AC, Tigli RS, Gumusderelioglu M, Nohutcu RM. bFGF-loaded HA-chitosan: a promising scaffold for periodontal tissue engineering. J Biomed Mater Res A. 2010;92(3):953-962.

3. Wu C, Zhou, Y, Lin C, Chang J, Xiao Y. Strontium-containing mesoporous bioactive glass scaffolds with improved osteogenic/ cementogenic differentiation of periodontal ligament cells for periodontal tissue engineering. Acta Biomater. 2012;8(10):3805-3815.

4. Duan X, Tu Q, Zhang J, et al. Application of induced pluripotent stem (iPS) cells in periodontal tissue regeneration. J Cell Physiol. 2011;226(1): $150-157$.
5. Jiong $\mathrm{C}$, Jiake $\mathrm{C}$, Chunmao $\mathrm{H}$, et al. Clinical application and long-term follow-up study of porcine acellular dermal matrix combined with autoskin grafting. J Burn Care Res. 2010;31(2):280-285.

6. Zhao $\mathrm{H}$, Wang $\mathrm{G}, \mathrm{Hu} \mathrm{S}$, et al. In vitro biomimetic construction of hydroxyapatite-porcine acellular dermal matrix composite scaffold for MC3T3-E1 preosteoblast culture. Tissue Eng Part A. 2011;17(5-6): 765-776.

7. Stevens MM, George JH. Exploring and engineering the cell surface interface. Science. 2005;310(5751):1135-1138.

8. Dalby MJ, Gadegaard N, Tare R, et al. The control of human mesenchymal cell differentiation using nanoscale symmetry and disorder. Nat Mater. 2007;6(12):997-1003.

9. Wang G, Zheng L, Zhao H, et al. In vitro assessment of the differentiation potential of bone marrow-derived mesenchymal stem cells on genipin-chitosan conjugation scaffold with surface hydroxyapatite nanostructure for bone tissue engineering. Tissue Eng Part A. 2011;17(9-10):1341-1349.

10. Liao F, Chen Y, Li Z, et al. A novel bioactive three-dimensional $\beta$-tricalcium phosphate/chitosan scaffold for periodontal tissue engineering. J Mater Sci Mater Med. 2010;21(2):489-496.

11. Tour G, Wendel M, Moll G, Tcacencu I. Bone repair using periodontal ligament progenitor cell-seeded constructs. J Dent Res. 2012;91(8): 789-794.

12. Cheng Z, Guo C, Dong W, He FM, Zhao SF, Yang GL. Effect of thin nano-hydroxyapatite coating on implant osseointegration in ovariectomized rats. Oral Surg Oral Med Oral Pathol Oral Radiol. 2012;113(3):e48-e53.

13. Guo J, Wang Y, Cao C, Dziak R, Preston B, Guan G. Human periodontal ligament cells reaction on a novel hydroxyapatite-collagen scaffold. Dent Traumatol. 2013;29(2):103-109.

14. Ge S, Zhao N, Wang L, et al. Bone repair by periodontal ligament stem cell-seeded nanohydroxyapatite-chitosan scaffold. Int J Nanomedicine. 2012;7:5405-5414.

15. Simmons PJ, Torok-Storb B. Identification of stromal cell precursors in human bone marrow by a novel monoclonal antibody, STRO-1. Blood. 1991;78(1):55-62.

16. Nehrer S, Breinan HA, Ramappa A, et al. Matrix collagen type and pore size influence behavior of seeded canine chondrocytes. Biomaterials. 1997;18(11):769-776.

17. O'Brien FJ, Harley BA, Yannas IV, Gibson, LJ. The effect of pore size on cell adhesion in collagen-GAG scaffolds. Biomaterials. 2005; 26(4):433-441.

18. Beck GR Jr. Inorganic phosphate as a signaling molecule in osteoblast differentiation. J Cell Biochem. 2003;90(2):234-243.

19. Beck GR Jr, Moran E, Knecht N. Inorganic phosphate regulates multiple genes during osteoblast differentiation, including Nrf2. Exp Cell Res. 2003;288(2):288-300.

20. Dalby MJ, Biggs MJ, Gadegaard N, Kalna G, Wilkinson CD, Curtis AS. Nanotopographical stimulation of mechanotransduction and changes in interphase centromere positioning. J Cell Biochem. 2007; 100(2):326-338.

21. Yim EK, Darling EM, Kulangara K, Guilak F, Leong KW. Nanotopography-induced changes in focal adhesions, cytoskeletal organization, and mechanical properties of human mesenchymal stem cells. Biomaterials. 2010;31(6):1299-1306.

22. Byers BA, Garcia AJ. Exogenous Runx2 expression enhances in vitro osteoblastic differentiation and mineralization in primary bone marrow stromal cells. Tissue Eng. 2004;10(11-12):1623-1632.

23. Sasano Y, Zhu JX, Kamakura S, Kusunoki S, Mizoguchi I, Kagayama M. Expression of major bone extracellular matrix proteins during embryonic osteogenesis in rat mandibles. Anat Embryol (Berl). 2000;202(1):31-37.

24. Zaidi SK, Javed A, Choi JY, et al. A specific targeting signal directs Runx2/Cbfa1 to subnuclear domains and contributes to transactivation of the osteocalcin gene. J Cell Sci. 2001;114(Pt 17): 3093-3102. 
International Journal of Nanomedicine

Dovepress

\section{Publish your work in this journal}

The International Journal of Nanomedicine is an international, peerreviewed journal focusing on the application of nanotechnology in diagnostics, therapeutics, and drug delivery systems throughou the biomedical field. This journal is indexed on PubMed Central, MedLine, CAS, SciSearch ${ }^{\circledR}$, Current Contents ${ }^{\circledR} /$ Clinical Medicine,
Journal Citation Reports/Science Edition, EMBase, Scopus and the Elsevier Bibliographic databases. The manuscript management system is completely online and includes a very quick and fair peer-review system, which is all easy to use. Visit http://www.dovepress.com/ testimonials.php to read real quotes from published authors.

Submit your manuscript here: http://www.dovepress.com/international-journal-of-nanomedicine-journal 